\title{
Link prediction in author collaboration network based on BP neural network
}

\author{
Chaoqun Chen ${ }^{1,2}$,Sanhong Deng ${ }^{1,2}$, Jing $\mathrm{Lu}^{3}$ \\ ${ }^{1}$ School of Information Management, Nanjing University, Nanjing, 210023, China \\ ${ }^{2}$ Jiangsu Key Lab of Data Engineering and Knowledge Service, Nanjing, 210023, China \\ ${ }^{3}$ Jiangsu Institute of Geological Survey, Nanjing, 210018, China
}

\begin{abstract}
Recently, more and more authors have been encouraged for collaboration because it often produces good results. However, the author collaboration network contains experts in various research directions within various fields, and it is difficult for individual authors to decide which authors are best suited to their expertise. This paper uses the relationships among authors to predict new relationships that may arise, recommending each author with the collaborators they may be interested in. The data source comes from 4-year data in DBLP from 2001 to 2004. After data cleaning, the training set and test set are constructed and then used BP neural network to build model. At the same time, this article compares the performance with Logistic Regression, SVM and Random Forest. The experiment shows that the BP neural network can get better result, and it is feasible to predict links in the author collaboration network.
\end{abstract}

\section{INTRODUCTION}

Link prediction is a very important research direction in social networks, such as Facebook, Twitter and Flicker and other social platforms, which recommend the friends to their users through link prediction. A link may mean a friend relationship in a social network, or it may mean a collaboration relationship in the collaboration network, or it may mean interaction in a protein network. Any kind of relationship between two nodes in a network can be considered a link. A definition of link prediction: Given a snapshot map of a social network at a time $\mathrm{G}=<\mathrm{V}, \mathrm{E}>$ and node $\mathrm{V}_{i}, \mathrm{~V}_{j}$, predict the probability of a link between nodes $V_{i}$, and $V_{j}$ [1]. It can be seen that the task of link prediction is divided into two categories: one is to predict the link that will appear in the future time, the second one is to predict the hidden unknown link in the space, this article discusses the former.

Link prediction has two main approaches: a score-based approach and a machine learning approach. The score-based approach is to consider link predictions as a regression problem by calculating the similarity scores for each pair of nodes and then sorting them, the order determines the likelihood of forming future links. The score-based approach is a simple and effective method, but this approach is sensitive to different features' weights. The machine learning approach is effective to use a variety of attributes to predict the formation of links, and does not need to give the weight of each feature manually, and it is easy to expand. Therefore, the method of machine learning has been widely used in link prediction. The machine learning approach regards the link prediction problem as a

\footnotetext{
${ }^{*}$ Corresponding author: $\underline{\text { sanhong } @ \text {, nju.edu.cn }}$
} 
classification problem, and then uses the SVM, decision tree, Boosting, Logistic regression and other methods [2].

Neural network (NN) is a powerful machine learning approach for nonlinear prediction, composed of independent units of neurons. Each processing unit sums the weighted inputs, and then applies the result to linear or non-linear functions to determine the outputs. In the neural network algorithm, BP neural network (Backpropagation network) has been very concerned about, with a strong non-linear mapping capabilities. BP neural networks have been widely used in various fields in recent years, including the prediction of the quality or robustness of plastic parts based on key process variables and material grade changes [3]. Besides, the use of BP neural networks for accurate shortterm load forecasting (STLF) has played an important role in the national and regional power system management [4]. However, in the field of link prediction, few scholars use the neural network algorithm, so the author will apply BP neural network to predict new links in author collaboration network, then explore whether it can achieve good results in this field.

\section{THE BASIC KNOWLEDGE ON BP NEURAL NETWORK}

This article uses a typical three-layer BP neural network, including input layer, hidden layer and output layer. The layers are full connected with edges and each edge corresponds to a weight w. At the same time, in addition to the neuron in input layer, neurons in other layers also include a bias $b$, those neurons will have an input value $z$ which received from weighted summation and an output value which received from nonlinearity transform $\mathrm{z}$ through the activation function. The activation function used in this article is the Sigmoid function $\left(\mathrm{f}(x)=\frac{1}{1+e^{-x}}\right)$. The input value of the neuron $\mathrm{j}$ in the first-layer is expressed as: $z_{j}^{l}=\sum_{i=1,2, \ldots, n} w_{i j} \cdot a_{i j}^{l-1}-b_{j}^{l}$.The variable i and the variable $\mathrm{j}$ represent neurons. ij represents the connection from the neuron $\mathrm{i}$ to neuron $\mathrm{j}, w_{i j}$ means the weight of the connection. $a_{i j}^{l-1}$ represents the output of the neuron $i$ in the 1-1 layer, that is, the $\mathrm{i}$-th input of the neuron $\mathrm{j}$ in the 1 layer. $b_{j}^{l}$ represents the bias of the neuron $\mathrm{j}$ in the 1 layer. Then the output of the neuron $\mathrm{j}$ in the 1 layer is expressed as $: a_{j}^{l}=\mathrm{f}\left(z_{j}^{l}\right)=\frac{1}{1+e^{-z_{j}^{l}}}$.

In practice, the $\mathrm{BP}$ neural network needs artificially specify the number of layers and the number of neurons in each layer. At the same time, the BP neural network needs to initialize the weight and bias of each edge in the initial stage and then the BP neural network algorithm will perform the forward transmission to get the predictive value for each sample of the training set. Then, according to the error value between the true value and the predicted value, the weight value of each connecting edge and the bias value of each layer in the neural network will be updated by backpropagation. The stopping conditions can be one of the following: a. The iterations times reach the given value; $b$. The error value is less than a given threshold. In this article, the stopping condition is to reach the given value of iterations times, that is, the number of times the parameter was updated reaches the given value.

Assuming that a given training set contains $\mathrm{m}$ training samples, we can use the gradient descent algorithm to solve the BP neural network. For example, for a training sample $(\mathrm{x}, \mathrm{y})$, define the cost function (the difference between the predicted value and the true value) is: $\mathrm{J}(W, b ; x, y)=\frac{1}{2}\left\|h_{W, b}(x)-y\right\|^{2}, \quad$ the $\operatorname{cost}$ function on the training set is: $\mathrm{J}(W, b)=$ $\left[\frac{1}{m} J\left(W, b ; x^{(i)}, y^{(i)}\right)\right]+$ $\frac{\lambda}{2} \sum_{l=1}^{n_{l}-1} \sum_{i=1}^{s_{l}} \sum_{j=1}^{s_{l}+1}\left(W_{j i}^{(l)}\right)^{2}$. It consists two parts: The first part is the sum of error mean square and the latter part is the regularization, which mainly 
to prevent the overfitting. In this article, the link prediction problem is transformed into a classification problem. The y value represents 0 or 1 , and $h_{W, b}(x)$ is the range of $[0,1]$ of the sigmoid function. The optimization problem of BP neural network is to obtain the $\mathrm{W}, \mathrm{b}$ that minimizes the cost function. In general, random values near 0 are used to initialize weights $\mathrm{W}$ and bias $b$, and then the value of $\mathrm{W}$ and $\mathrm{b}$ is updated by optimization algorithm such as gradient descent $\quad$ algorithm: $\quad W_{i j}^{(l)}=W_{i j}^{(l)}-$ $\alpha \frac{\partial J(W, b)}{\partial W_{i j}^{(l)}}, b_{i}^{(l)}=b_{i}^{(l)}-\alpha \frac{\partial \mathrm{J}(W, b)}{\partial b_{i}^{(l)}}$. The new cost function value can be obtained with the new $\mathrm{W}$ and $b$ values and the cost function value will keep close to its minimum value gradually. In the above formula, $\alpha$ indicates the learning rate which can be understood as the pace of each gradient, that is, the decreasing rate of cost function. Its value is very important. If the value is oversized, the decreasing rate may be too fast and missed the minimum value of cost function. If the value is too small, the cost function may be converged very slowly. The general $\alpha$ value is between 0.001-0.3. At the same time, it can be seen that when the sample size is relatively large, if updated value $\mathrm{W}$ and $\mathrm{b}$ by all the data set each time, the efficiency will be relatively low. Therefore, this article uses Stochastic Gradient Descent (SGD), and each iteration process just selects a part of dataset randomly.

In order to evaluate the performance of BP neural network in the author's collaboration network linkage prediction, this paper compares the results of the three algorithms: Logistic Regression, SVM and Random Forest.

\section{EXPERIMENTAL AND ANALYSIS}

\section{RESULTS}

\subsection{Dataset}

The dataset used in this article is an open social network dataset DBLP (Digital Bibliography and
Library Project) which is an integrated database system of English literature taking the author as the core on research results in the computer science field. The author's research achievements, including published papers in international journals and conferences, are listed according to the year. The data set of DBLP is very large. Considering the time cost, the author only experimented with data from 2001 to 2004, and removed the data which only has one author. Divided the four-year data into two periods, 2001-2002 and 2003-2004, this article constructed the features by 2001-2002 data and the labels by 2003-2004 data. If the authors have no collaboration relationship in 2001-2002 while they have the partnership in 2003-2004, the label is $y=1$, which indicating that a new link appears between the author's pair. Otherwise the label is $\mathrm{y}=0$, which indicating that no new links appear between the authors. It is noteworthy that, during the feature construction, the selected author's pair should have no collaboration relationship in 2001-2002, and then to predict whether they would collaborate in 2003 or 2004 . At the same time, the author notes that some authors are active only in 2001-2002 and no longer appear in 20032004; Conversely, some authors are active only in 2003-2004 while no longer appear in 2001-2002. In the author collaboration network, it will result in the mismatched between features and labels. To avoid this situation, this article considers only the authors that are included both in the two time periods. At the same time, due to the fact that the number of $\mathrm{y}=0$ is far greater than $\mathrm{y}=1$, namely the imbalanced learning problem. Therefore, in the experiment, the author randomly selected minority examples in the negative samples (the samples which $\mathrm{y}=0$ ), this article obtains the negative samples which has the same quantity with positive samples (the samples which $y=1$ ), both are 5,504 samples. As a result, the social network constructed in this article is an undirected graph without weight with 8,363 nodes in the network. In the process of splitting 
training set and test set, 70\% samples are randomly divided as training sets and the remaining $30 \%$ are test sets, in which there are 7,707 samples in training set, and 3,302 samples of test sets.
For the feature vector, the author refers to the researches of other scholars [5 7] and selects the following features as shown in Figure 1, these features are derived from the author collaboration network where each node represents an author:

\subsection{Features}

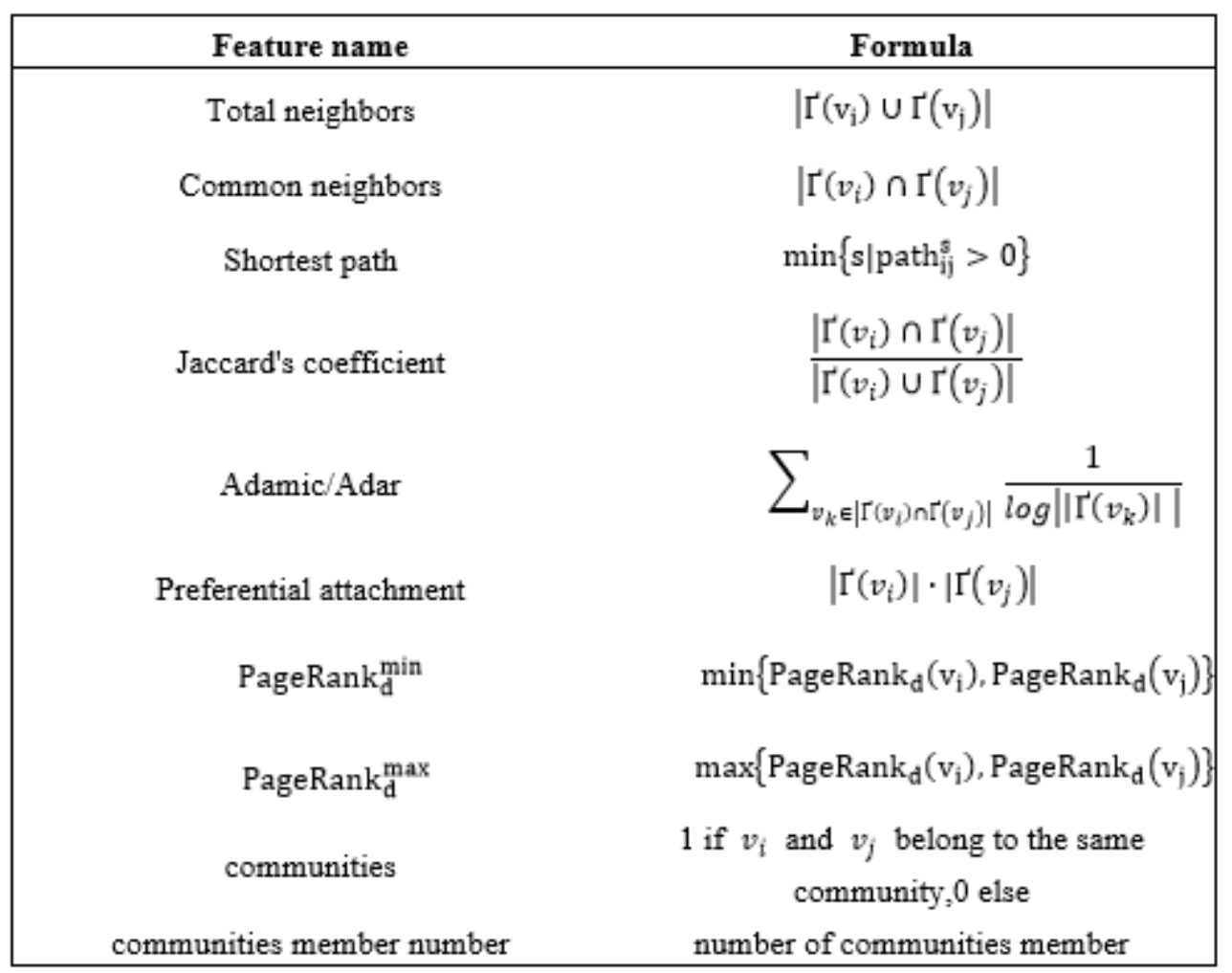

Fig1. Features computed for each author pair $\left(\mathrm{V}_{i}, \mathrm{~V}_{j}\right)$

The Total neighbors of the two nodes $v_{i}$ and $v_{j}$ is defined as the total numbers of their neighbors, and the common neighbor calculates only once. Common neighbors calculate the number of common neighbors that two nodes have. For example, for two authors of a collaboration network, this is the number of other authors they have worked together. Shortest path is the minimum number of edges connected with two nodes. If there is no such connection path, this feature value is usually considered to be infinity. Jaccard's coefficient [8] is the normalized metric for Common neighbors, which calculates the proportion of the two authors common neighbors in their all neighborhoods. This feature sometimes has better performance than Common neighbors, especially when the number of one node's neighbors is much greater than the other node's. Adamic/Adar [9] measures the similarity of two nodes by assigning weights to common neighbors. The basic principle is that the smaller the neighbors number of the common neighbor, the greater the contribution to the similarity, that is to say, the more likely the two nodes are to be linked. Preferential attachment [10] indicates that a node with a higher centrality degree is more likely to produce new links. PageRank is the core algorithm used by Google to rank search results [11]. The PageRank value of webpage $v_{i}$ indicated by formula: PageRank $k_{d}\left(v_{i}\right)=(1-$ 
$d)+d \sum_{v_{m} \in \Gamma\left(v_{i}\right)} \frac{\operatorname{PageRank}_{d}\left(v_{m}\right)}{\Gamma\left(v_{m}\right)} \quad$,where $\Gamma\left(v_{i}\right)$ refers to the webpages link to webpage $v_{i}$. In fact, in the graph structure, the PageRank value of a node is a probability used to represent the likelihood that a person randomly walking on edges will arrive at any particular node. $d$ is a damping factor which mainly considering that there are some nodes in the directed graph do not point to other nodes, which may result in a dead loop when accessing this node. Therefore, it is necessary to modify the PageRank formula, that is, to add the damping factor. The value is 0.85 in general. In this article, PageRank is used for the undirected graph. $\Gamma\left(v_{i}\right)$ refers to all the neighbor nodes of the node. Because the authors pair is considered, the larger PageRank value between two authors is expressed as pagerank ${ }_{d}^{\max }$ while the smaller PageRank value is pagerank ${ }_{d}^{\min }$. Many complex networks can be divided into communities, and the nodes in the same community are more closely linked. For authors collaboration networks, authors in the same community are more likely to have partnerships. This feature of the Communities is used to indicate whether the two authors are belonging to the same community. This article uses the clique percolation method in Python to discover community [12]. For authors in the same community, the communities' member number represents the number of authors in the community. Generally speaking, the smaller the number of authors in a community, the more likely that two authors in this community will collaborate.

\subsection{Performance metrics}

The performance metrics of this article adopts to evaluate and compare algorithms are precision, recall and F1 rates. Several terms must be identified before introducing specific indicators: TP represents true-positive which means a positive sample is predicted as positive by the model; FN represents false-negative when a positive sample is predicted as negative wrongly by the model; In the negative case, if a sample is predicted correctly, the prediction is said to be true-negative (TN);otherwise it is falsepositive(FP). P represents the positive sample, namely there is a collaboration relationship between authors. $\mathrm{N}$ represents a negative sample, namely there is no collaboration relationship between authors. Precision is expressed by formula: $\frac{|T P|}{|T P|+|F P|}$; Recall is $\frac{|T P|}{|T P|+|F N|}$ and the F rate is $\frac{1}{\partial \frac{1}{\text { precision }}+(1-\partial) \frac{1}{\text { recall }}}$. The $\partial$ is between 0 and 1 . When $\partial=0.5$, it indicates that the precision and recall rate are equally important; when $\partial>0.5$, it indicates that precision is more important; in this paper, the precision and recall rate are treated as the same important, that is, $\partial=0.5$, and the $\mathrm{F}$ rate is called $\mathrm{F} 1$ rate at this time.

\subsection{Experient result}

In the experiment, the number of neurons in the input layer, hidden layer and output layer of BP neural network were 10,50 and 2 . In practice, the iterations times epoch and the learning rate eta have an effect on the performance of BP neural network. This article has carried on several experiments. Under the condition of epoch $=200$, this article respectively chooses the learning rate including $0.001,0.003,0.01,0.03,0.1$ to carry on the experiment. It can be found that the precision rate of the model on the test set is increasing, while the recall rate is decreasing because the model predicts part of negative samples as positive with the increase of learning rate (Fig 2). And in terms of F1, under the learning rate of 0.003 BP neural network can perform better. Therefore, the author choses the learning rate of 0.003 and does experience in the iterative times of 100, 200, 300, 400 and 500 to obtain BP neural network performance metrics under different iterations times. It can be seen that the recall rate of the model is at the maximum in the test set when the iteration number is 100 and the $\mathrm{F} 1$ rate 
at this time also gets the maximum value (Fig. 3). Therefore, we chose 100 as iteration times and 0.003 as the learning rate finally. At the same time, the BP neural network is compared with the Logical Regression, SVM and Random Forest in the same test set, and the result is shown in Figure 4. The recall rate of $\mathrm{BP}$ neural network is the highest, and the F1 rate is higher than Logistic Regression, which is approximately the same as the Random Forest.

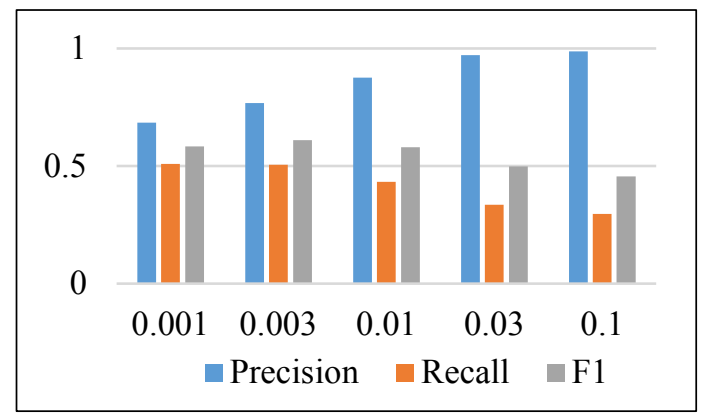

Fig. 2. BP neural network classification results on different learning rate $($ epoch $=200)$

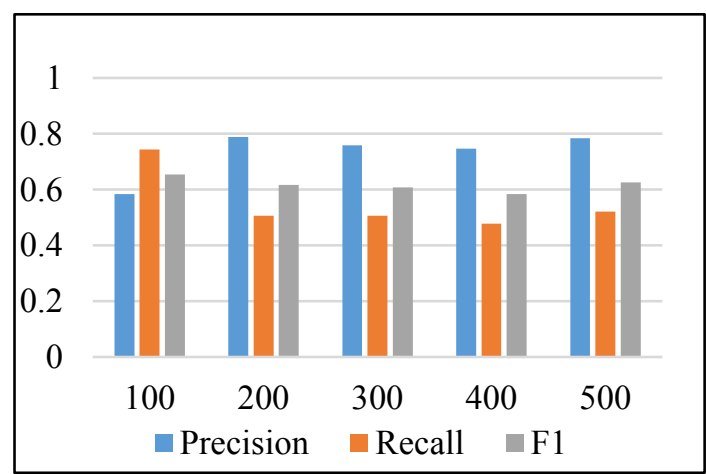

Fig. 3. BP neural network classification results on different iteration times(eta $=\mathbf{0 . 0 0 3})$

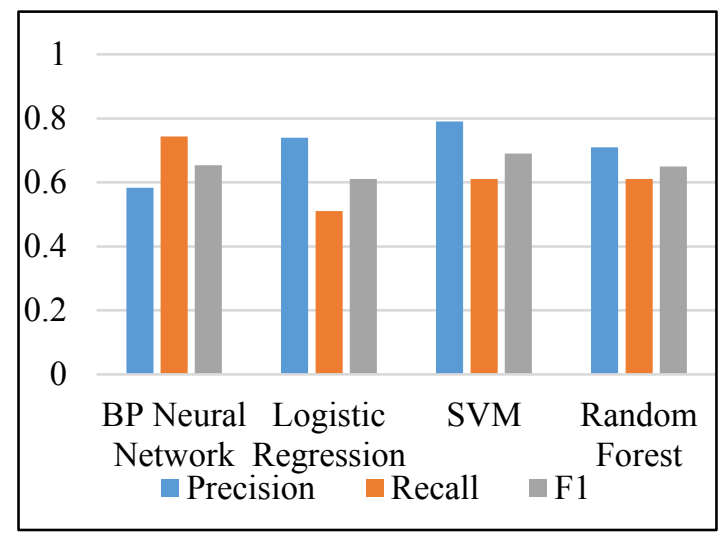

\section{References}

Fig. 4. Classification results for different algorithms

\section{Conclusion}

This article takes the data from 2001 to 2004 in DBLP as dataset and constructs 10 attributes as feature vector such as total number of neighbors, common neighbors and Adamic/Adar. Next, this article uses BP neural network to predict links in the author collaboration network and compares the performance metrics with Logistic Regression, SVM and Random Forest. Finally, it is found that the model trained by BP neural network outperforms other algorithms on recall rates, and the F1 rate is also good, namely, there is valuable information to be learned from the structure of the author collaboration network. Therefore, it is reasonable to apply BP neural network to link prediction in social network. Of course, there are deficiencies in this article. The first is that the features used to describe the structure of a collaboration network in the paper is not comprehensive, and some features those may affect authors' collaboration relationship may not take into account. The second is that the $\mathrm{F} 1$ rate is not particularly good and need to improve. In future, we can increase the accuracy of the predictor by adding the features, the number of the hidden layers and the number of neurons in BP neural network, and optimizing the parameter initialization.

This work has been supported by the Youth Project of the National Natural Science Foundation of China under the Grant Numbers 71503121.

1. H. YIN. Link Prediction in Social Network". Master's Thesis, Jilin University, Jilin, (2012). 
2. C. Ahmed, A. Elkorany, R. Bahgat. A supervised learning approach to link prediction in Twitter. Social Network Analysis \& Mining, 6, (2016), 1-11.

3. B.H.M. Sadeghi. A BP-neural network predictor model for plastic injection molding process. Journal of Materials Processing Technology, 103, (2000), 411-416.

4. Z. Xiao, S.J. Ye, B. Zhong, et al. BP neural network with rough set for short term load forecasting. Expert Systems with Applications, 39, (2009), 273-279.

5. D. Liben-Nowell, J. Kleinberg. The link prediction problem for social networks[J]. Journal of the Association for Information Science \& Technology, 58, (2007), 10191031.

6. Hasan, A. Mohammad. Link Prediction using Supervised Learning[J]. Proc of Sdm Workshop on Link Analysis Counterterrorism \& Security, 30, (2006), 798-805.
7. M. Pavlov, R. Ichise. Finding experts by link prediction in co-authorship networks. International Conference on Finding Experts on the Web with Semantics. CEUR-WS.org, (2007), 42-55.

8. G. Salton, M.J. McGill. Introduction to Modern Information Retrieval. Program, 55, (2004), 239-240, 2004.

9. L.A. Adamic, E. Adar. Friends and neighbors on the Web. Social Networks, 25, (2003), 211-230.

10. M.E.J. Newman. Clustering and preferential attachment in growing networks. Physical Review E Statistical Nonlinear \& Soft Matter Physics, 64, (2001), 025102.

11. S. Brin, L. Page. Reprint of: The anatomy of a large-scale hypertextual web search engine. Computer Networks, 56, (2012), 3825-3833.

12. G. Palla, I. Derényi, I. Farkas, et al. Uncovering the overlapping community structure of complex networks in nature and society. Nature, 435, (2005), 814. 